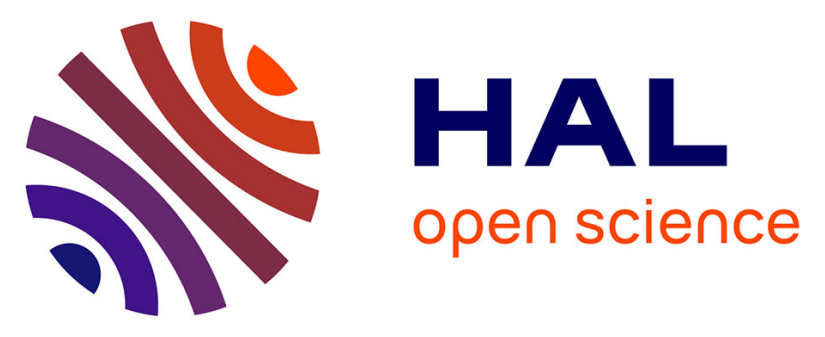

\title{
Towards a better understanding of pelvic system disorders using numerical simulation
}

Pauline Lecomte-Grosbras, Mouhamadou Nassirou - Diallo, Jean-François

Witz, Damien Marchal, Jérémie Dequidt, Stéphane Cotin, Michel Cosson, Christian Duriez, Mathias Brieu

\section{To cite this version:}

Pauline Lecomte-Grosbras, Mouhamadou Nassirou - Diallo, Jean-François Witz, Damien Marchal, Jérémie Dequidt, et al.. Towards a better understanding of pelvic system disorders using numerical simulation. Medical Image Computing and Computer Assisted Intervention, Sep 2013, Nagoya, Japan. hal-00831245

\section{HAL Id: hal-00831245 \\ https://hal.inria.fr/hal-00831245}

Submitted on 6 Dec 2013

HAL is a multi-disciplinary open access archive for the deposit and dissemination of scientific research documents, whether they are published or not. The documents may come from teaching and research institutions in France or abroad, or from public or private research centers.
L'archive ouverte pluridisciplinaire HAL, est destinée au dépôt et à la diffusion de documents scientifiques de niveau recherche, publiés ou non, émanant des établissements d'enseignement et de recherche français ou étrangers, des laboratoires publics ou privés. 


\title{
Towards a better understanding of pelvic system disorders using numerical simulation
}

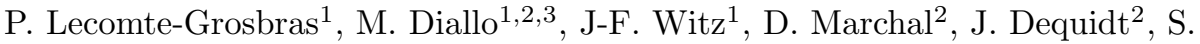 \\ $\operatorname{Cotin}^{2}$, M. Cosson ${ }^{3}$, C. Duriez ${ }^{2}$, M. Brieu ${ }^{1}$ \\ ${ }^{1}$ Laboratoire de Mécanique de Lille, École Centrale de Lille \\ ${ }^{2}$ INRIA - University of Lille $1{ }^{3}$ CHRU Lille - University of Lille 2
}

\begin{abstract}
Genital prolapse is a pathologic hyper-mobility of the organs that forms the pelvic system. Although this is common condition, the pathophysiology of this disorder is not well known. In order to improve the understanding of its origins, we recreate - virtually - this biomechanical pathology using numerical simulation. The approach builds on a finite element model with parameters measured on several fresh cadavers. The meshes are created from a MRI of a healthy woman and the simulation includes the mechanical interactions between organs (contacts, ligaments, adhesion...). The model is validated through comparison of functional mobilities of the pelvic system observed on a dynamic MRI. We then propose to modify, step by step, the model and its parameters to produce a pathologic situation and have a better understanding of the process. It is not a formal proof but the numerical experiments reinforce the clinical hypothesis on the multifactorial origins of the pathology.
\end{abstract}

\section{Introduction}

Genital prolapse is a pathologic hyper-mobility of the pelvic system's organs. It induces a strong discomfort and incontinence. This pathology affects one third of women, whatever the age [1] and more than $60 \%$ of women older than 60 [2]. To improve quality of life, surgical treatments exist with an intervention rate of $12 \%$ at the age of 80 . Unfortunately, these interventions have a failure of $30 \%$ independently of the surgical technic. The main reason of these failures relates to the complex and multifactorial physiopathology of the prolapse which is still not perfectly understood.

In order to better understand the physiological mobilities and to improve the surgical technics, several works have started to model the pelvic system. For instance, an important work on tissue characterization has been leaded, using destructive methods [3][4]. Other work address the problem of the evolution of soft connective tissue characteristics with respect to the age and/or the pathology [4] [5]. These results provide a range of tissue properties and evolution for several structures that can possibly be compared. But they can not provide a global understanding of the respective contribution of each element on the deformations of pelvic organs. Some previous work present FEM based models of the pelvic system. For instance, FEM model is used to simulate the bladder filling [7]. In 
[8], bladder filling is used to get the material properties by inverse simulation. In [9] a FEM approach completed by characterization measurements provides a first FEM model dedicated to pelvic system mobilities. In [11], the work is completed by a study of the ligaments modeling. The problem of modeling the interactions between the pelvic organ is faced in [6] but the solution is only 2D.

In this paper, we build on these works to provide a complete 3D FEM simulation with a wide variety of boundary conditions (contacts with friction, role of ligaments, adhesion and fixations), in order to reproduce the healthy pelvic mobilities that are validated with a dynamic MRI. But the main contribution of this work is to use and degrade the FEM model till progressively reaching a simulation of pathological cases. The topology of the ligament support system is also modified while carefully relying on anatomical and clinical references. We then compare the mobilities obtained in simulation to the ones observed clinically. Finally, the pathologic example of cystocele illustrates how this simulation tool provides new evidence on the phenomena at the origin of the disease.

\section{Bio-mechanical model of the pelvic system}

In order to build the FEM simulation of the pelvic system mobilities, it is necessary to have a geometrical model of the anatomy, the mechanical properties of tissues and a good modeling and numerical tools for the boundary conditions (attachments, loadings, contacts, etc.). This section describes how the physicsbased model of pelvic system mobilities is obtained.

Geometrical model : The geometry of the key structures of the pelvic system is obtained from segmentation of MR images of voluntary healthy women. The images are segmented to get the geometrical contours. The image is taken while the bladder is full in order to have a good contrast on the image. For the vagina and the rectum, a gel is inserted in order to increase the contrast. From the data

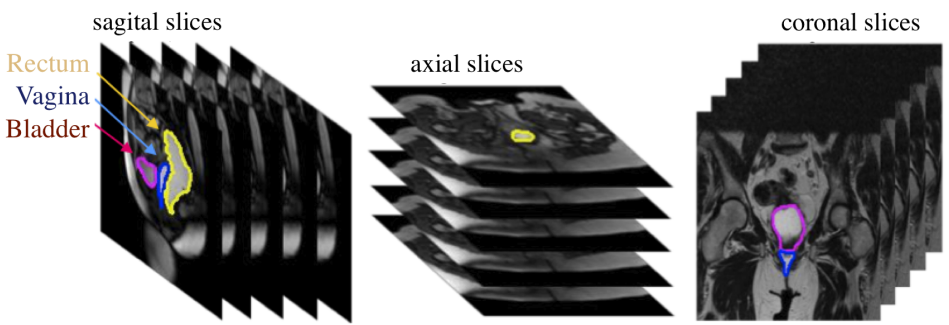

Fig. 1: Geometric model construction: contour segmentation.

we get also the contour of the uterus and the bony pelvis. However, the MRI does not provide enough signal for the segmentation of the ligament system of the pelvic floor. Its geometry was reconstructed according to anatomical data, available in the literature [12]. 

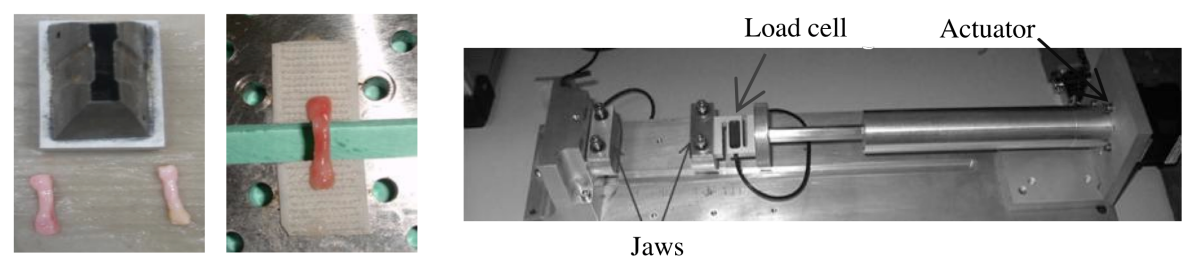

Fig. 2: Protocol and experimental set-up for the mechanical characterization of soft tissues. The samples are cut using a punch (left). Their geometry was defined in order to obtain a homogeneous deformation.

Mechanical properties of soft-tissues : Experimental measurements were conducted in order to estimate the mechanical properties of the tissues that form the pelvic system. The protocol used for ex vivo characterization has been carefully built (control of hygrometry of the tissue, body temperature, rate of deformation for visco-elasticity, etc.) [9] [10]. A portable uniaxial traction machine (see Figure 2) was developed and used in order to do the experiments at the morgue, on 18 fresh cadavers (without pathology) with a mean age of 75 . Several mechanical tests were realized on each cadaver (2 to 3 per organ) which makes a total of more than 300 tests. These tests are completed by measurements on pathologic tissues (see section 3) for a total of up to 2000 tests. Biaxial tension tests have been considered but would require too large samples for the considered organs (with our machine: $9 \mathrm{~cm} \times 9 \mathrm{~cm}$ ). Moreover, our experimental results reveal that there is no statistical evidences of anisotropy for pelvic soft tissues. In our measurements, if we sort the organs according to their stiffness, the order is always the same. The vagina is the organ made of the stiffer tissue, whereas the rectum and the bladder are softer. Even if the dispersion is important between cadavers, we can define - statistically - average mechanical properties for each organs if we define classes of population related to age and nulliparous or not. The following table provides the mechanical properties used for the tissues to build the healthy model:

\begin{tabular}{lcc}
\hline & Young Modulus & \\
Anatomical structure & median (interquartile range) & Thickness/Diameter $(\mathrm{mm})$ \\
\hline Rectum & $0,54(0.66) \mathrm{MPa}$ & 3 \\
Vagina & $0,67(2,22) \mathrm{MPa}$ & 4 \\
Bladder & $0,24(1.5) \mathrm{MPa}$ & 3 \\
Pelvic floor (quantitative data) & $3 \mathrm{MPa}$ & 10 \\
Uterosacral Ligament & $0,78(1.98) \mathrm{MPa}$ & 8 \\
Round Ligament & $1,32(1.08) \mathrm{MPa}$ & 4 \\
Large Ligament & $2.22(4.5) \mathrm{MPa}$ & 4 \\
\hline
\end{tabular}

For the simulation, we use the Finite Element code available in SOFA [13]. The organs of the pelvic system are mostly hollow, so for modeling their deformation, we use triangular elements based on shell theory (both membrane and bending energies are modeled). Due to its high rigidity, the uterus is modeled as a rigid body. For modeling the ligaments, series of beam elements are used. The 
tissues are considered as almost incompressible due to their high level of water content. Thus we use a Poisson ratio of 0.45 for deformable shells.

Boundary conditions : At this stage, the model includes the organ geometry and their mechanical properties, but the boundary conditions play a central role in the resulting mobilities. The suspension and the support systems of the organ are needed, as well as the modeling of the interactions between the organs.

For the ligaments, the literature often highlights the role of the round, large and uterosacral ligaments. This is in line with our observations during the dissection and measuring programs on cadavers. But, to obtain better results on the mobilities, we have also modeled some existing ligaments that are less documented: paravaginal and umbilical. The role of the paravaginal ligament is fundamental to limit the retroversion of the uterus and the umbilical ligament limits the mobility at the top of the bladder. The figure 3 presents two views on the models with the organs that are modeled and the ligaments.

The analysis of the displacement field of a dynamic MRI shows that there is two zones where the organs are fixed: where the bladder is in contact with the pubis, and where the rectum is in contact with the sacrum. These adhesions are modeled using fixed boundary conditions on the models. Finally, in order to model the mechanical interactions between the organs and reproduce the role played by the facias, we use a friction contact model, as proposed in 2D in [6]. The model relies on Signorini conditions and Coulomb friction law and leads to complex non-smooth dissipative behaviors. We benefit from the numerical treatment of such unilateral contact constraints that is provided by SOFA [13].
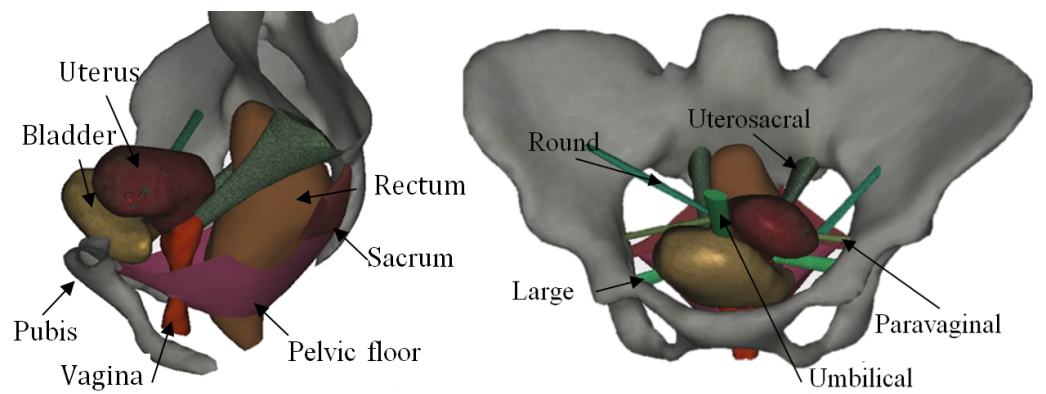

Fig. 3: Complete model of the pelvic system: the four organs, the pelvic floor, the umbilical ligament and the four symmetric pairs of ligaments: round, paravaginal, large and uterosacral.

Validation for healthy tissues : The figure 4 shows a comparison between the displacement that are measured by dynamic MRI of a healthy woman. The displacements are obtained when simulating a push effort. The data in [12] indicates that such effort can be modeled by a pressure $P$, in the order of $10 \mathrm{MPa}$, oriented at a 45 degree angle. This is a strong hypothesis that is very difficult to verify, yet the difference between the measured displacements and simulated ones is less than $1 \mathrm{~cm}$ while the maximal displacements is more than $10 \mathrm{~cm}$. 

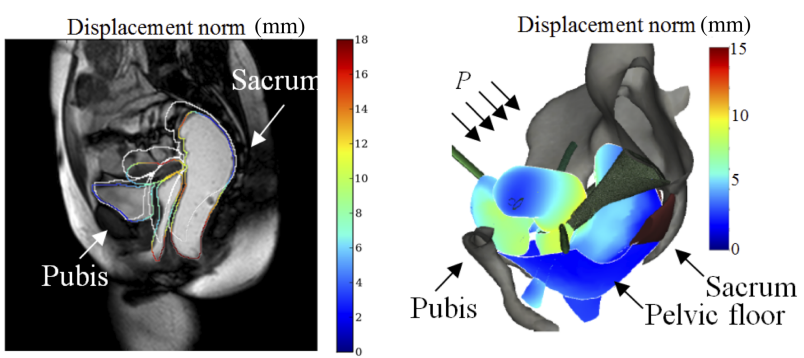

Fig. 4: The displacements of the organs are measured on a dynamic MRI to validate the displacements obtained by FEM simulation on healthy woman.

\section{Simulation of the multi-factorial origins of pathologies}

According to mechanical studies, pelvic sagging pathologies are mostly due to the elongation of the ligaments but other factors like the change of tissues properties, and the degradation of the fascias are also mentioned. However, in real life, it is impossible to evaluate separately the impact of each of these factors. Yet it is a relevant information for reparative surgery. In this work, we propose a first answer based on a set of numerical experiences, on the healthy biomechanical model of the pelvic system. These experiences illustrate the role that the different factors can play in the pathology.

The role of ligaments : For obtaining healthy physiological mobilities, we have placed, modeled and use several ligaments. If we refer to anatomical books, and in particular to comparative anatomy [14], it can be seen that all female mammals have round, large, paravaginal and umbilical but only biped mammal have uterosacral ligaments. In order to understand the reason, we have realized some simulation in which the direction of the gravity changes compared to the body axis. Figure 5 shows the force exerted on the ligaments compared to the direction of gravity: it highlights the role of the ligaments according to the body angle.

These simulations allow to confirm that in the lifted position $\left(\theta<35^{\circ}\right)$ the uterosacral ligaments play a predominant role, while in a lying position, the

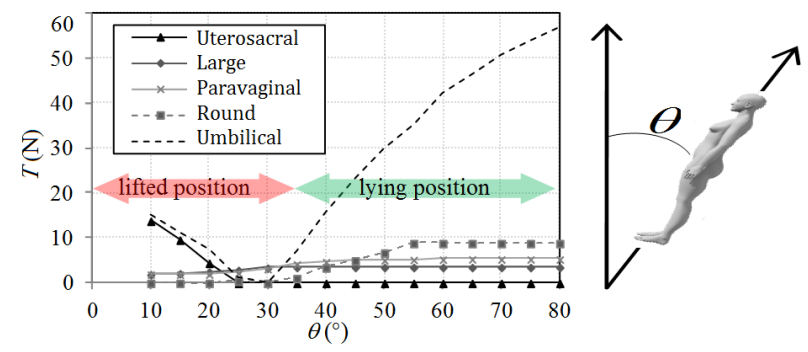

Fig. 5: Forces in the ligaments according to different position of the body. Calculated by simulation. 

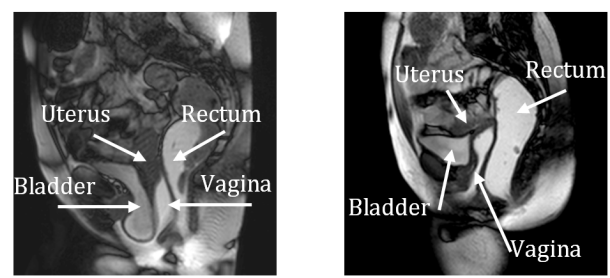

Fig. 6: Left: Dynamic MRI of a cystocele pathology, Right Dynamic MRI of healthy tissues. The motion of the bladder is completely different.

contribution of the paravaginal and umbilical ligaments is crucial. These results allow to understand why important work published in pelvic surgery studies consider empirically, that the uterosacral ligaments play a key role on the pelvic mobilities.

Modeling the prolapse pathology : The following simulations consist in degrading progressively the healthy physiological model in order recreate, artificially, a frequent pathological mobility: the cystocele. The cystocele is a pathology that leads abnormally important motion of the bladder that can lead to an important sagging of the vagina and the bladder and to incontinence problems. Figure 6 (left) shows a MRI issued from a dynamic sequence in the sagittal plane in the case of a cystocele pathology. During the push effort, the bladder undergoes an important displacement and tends to move out of the body. This pathologic mobility can be compared with healthy mobilities during the same effort (Figure 6 right).

In order to identify the role of each element in the pathology, we intentionally degrade the functional healthy model in order to reproduce the pathologic motion of the bladder. Several steps were used to degrade the model. We conducted additional experimental measures of characterization using biological tissues taken during prolapse surgeries (issued from about 40 patients) and also on 5 pathologic fresh cadavers (approximatively the same age than the nonpathologic ones). These experiments highlight the discrepancies in the characteristics of pathological and healthy tissues. Paradoxically, contrary to what we might expect, the pathologic tissues are often stiffer than the healthy ones (see
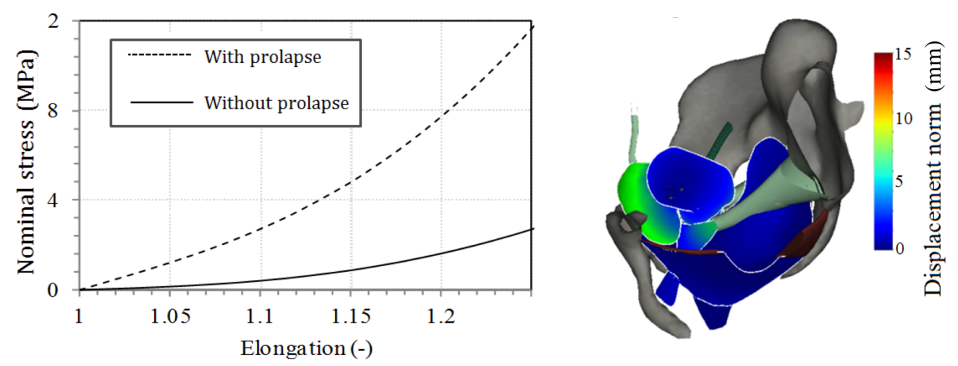

Fig. 7: Comparison of the mechanical behavior of vagina tissues of cadaver, with and without genital prolapse - Simulation obtained using pathologic constitutive law. 

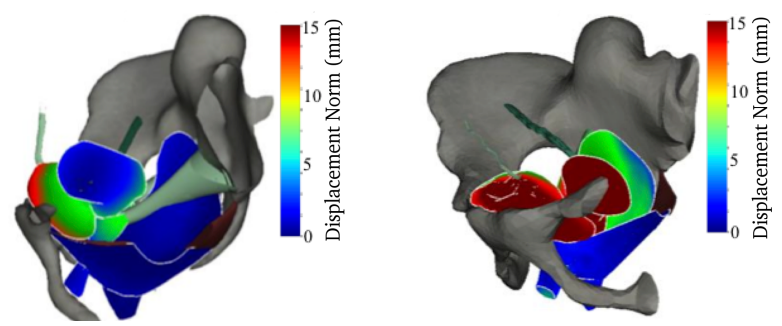

Fig. 8: Simulation of the elongation of the ligaments (left), elongation of ligaments and degradation of fascia between bladder and pubic bone.

Figure 7 left).The simulation of the mobilities while using the properties of the pathologic tissues is not sufficient to reproduce a cystocele by simulation (Figure 7 right).

It is often considered that the cystocele is due to an elongation of ligaments, induced by micro damages cumulated through the years. We progressively augment the length of the uterosacral ligament in order to get, on the biomechanical model, the same level of displacement that are observed for the cervix on pathological data. We obtain an increasing of the length of about $10 \%$. However, we notice that the mobilities are not sufficient to re-create, by simulation, a cystocele. We then applied successively the $10 \%$ augmentation of the length for all ligaments (especially the umbilical ligament in the case of the bladder) and even a complete rupture of umbilical ligament but the obtained displacements were not sufficient to be considered as pathologic.

In fact, an other factor of cystocele is the degradation of the adhesions of the bladder on the pubis, that are considered as perfect in healthy situations. The simulation presented on the right of figure 8 , is obtained by combining a $10 \%$ elongation of the ligaments and the degradation of the mechanical properties of the fascias between the bladder and the public bone. The perfect adhesion is replaced by a friction contact law combined with an elastic link with a low stiffness. The obtained mobilities for the cervix and the bladder now corresponds to the ones that can be observed during a cystocele.

Discussion: The successive degradation of the biomechanical model of the pelvic system mobilities allows to switch from healthy mobilities to pathological mobilities. The simulation reinforce the hypothesis of a multi factorial origin for the pathology for cystocele: it seems to be due to a change of traumatic elongation of the ligaments and to a degradation of the adhesive fascias. Of course, the impact of these results is limited by the fact that we can not validate the simulations of the pathology by ground truth data. Indeed, these data do not exist as we have created artificially a pathology starting from a healthy set of data!

\section{Conclusion and perspective}

This paper presents a simulation tool of the pelvic system that is based on characterization of the tissues, modeling the boundary conditions and providing Finite 
Element approach. The main contribution is to provide an assessment, thanks to numerical experiments, to the hypothesis made by the medical community on the origin of the cystocele. In future work, we plan to work on patient-specific models based on a fusion of 3D images and 2D dynamic data with our simulation. From pathologic mobilities observed in the images, we will try to identify the main factors that induce the pathologic mobilities and adapt the parameters of our simulation accordingly. Then, we could then compare the results with experimental ground truth data collected during reparative surgery and validate this numerical simulation tool. If predictive, we could then use it for individual planning of surgery.

\section{References}

1. Samuelsson E., Victor F., Tibblin G., Svardsudd K.: Signs of genital prolapse in a Swedish population of women 20 to 59 years of age and possible related factors. Am. J. Obstet. Gynecol., Vol. 180, 299-305 (1999).

2. Swift S.: The distribution of pelvis organ support in a population of female subjects seen for routine gynecologic health care. Am. J. Obstet. Gynecol., Vol. 183, 277-285 (2000).

3. Ettema G.J., Goh J.T., Forwood M.R.: A new method to measure elastic properties of plastic-viscoelastic connective tissue. Med Eng Phys, Vol. 20, 308-314 (1998).

4. Goh J.T.: Biomechanical properties of prolapsed vaginal tissue in pre and postmenopausal women. Int Urogynecol J Pelvic Floor Dysfunct, Vol. 13, 76-79 (2002).

5. Gilchrist AS., Gupta A., Eberhart RC., Zimmern PE.: Biomechanical properties of anterior vaginal wall prolapse tissue predict outcome of surgical repair. J. Urol., Vol. 183.3, 1069-1073, (2010).

6. Chen Z.W., Joli P., Feng Z.-Q.: Finite element modeling of interactions between pelvic organs due to pressure. CSMA (2011).

7. Krywonos J. et al.: MRI image-based FE modelling of the pelvis system and bladder filling. Comput. Method. Biomec., Vol. 13.6, 669-676 (2010).

8. Balakrishna H. et al.: PelvicSim-A Computational-Experimental System for Biomechanical Evaluation of Female Pelvic Floor Organ Disorders and Associated Minimally Invasive Interventions. Stud Health Technol Inform, Vol. 119, 182 (2005).

9. Rao GV., Rubod C., Brieu M., Bhatnagar N., Cosson M.: Experiments and finite element modeling for the study of prolapsed in the pelvic system. Comput. Method. Biomec., Vol. 13.3, 349-357 (2010).

10. Rubod C., Brieu M., Cosson M., Rivaux G., Clay JC., Gabriel B.: Biomechanical properties of human pelvic organs, Urol., Vol. 79.4, 1346-1354 (2012).

11. Vallet A., Witz JF., Brieu M., Rubod C., Cosson M.: Simulation of pelvic mobility: topology optimization of ligamentous system. Comput. Method. Biomec., Vol. 14.1, 159-163 (2011).

12. Kamina P. Clinical Anatomy, book number 4, Maloine edition (2008).

13. Faure F. et al. SOFA: A Multi-Model Framework for Interactive Physical Simulation. Soft Tissue Biomechanical Modeling for Computer Assisted Surgery, 283-321 (2012), http://www.sofa-framework.org.

14. Baronne R.: Comparative anatomy of domestic mammals, Vigot edition (2009). 